\section{Toward Noncooperative Iris Recognition: A Classification Approach Using Multiple Signatures}

\author{
Hugo Proença and Luís A. Alexandre
}

\begin{abstract}
This paper focus on noncooperative iris recognition, i.e., the capture of iris images at large distances, under less controlled lighting conditions, and without active participation of the subjects. This increases the probability of capturing very heterogeneous images (regarding focus, contrast, or brightness) and with several noise factors (iris obstructions and reflections). Current iris recognition systems are unable to deal with noisy data and substantially increase their error rates, especially the false rejections, in these conditions. We propose an iris classification method that divides the segmented and normalized iris image into six regions, makes an independent feature extraction and comparison for each region, and combines each of the dissimilarity values through a classification rule. Experiments show a substantial decrease, higher than 40 percent, of the false rejection rates in the recognition of noisy iris images.
\end{abstract}

Index Terms-Iris classification, noncooperative iris recognition, biometrics.

\section{INTRODUCTION}

SINCE 1987, when Flom and Safir [1] observed the stability of iris morphology over human lifetime and estimated the probability for the existence of two similar irises at 1 in $10^{72}$, the use of iris biometrics has been increasingly encouraged by both government and private entities. The iris is commonly recognized as one of the most reliable biometric traits: It has a random morphogenesis and, apparently, no genetic penetrance.

Under rigid image capturing constraints, it is possible to obtain good quality images and achieve impressive accuracy with very low error rates. However, these error rates substantially increase, especially the false rejections, when the images do not have enough quality, either due to focus, contrast, or brightness problems and iris obstructions or reflections. This is a problem commonly identified by several authors (e.g., [2], [3], [4]).

For the sake of accuracy, present iris recognition systems require that the subjects stand close (less than 2 meters) to the imaging camera and look for at least 3 seconds, until the required data is captured [5]. This cooperative behavior is regarded as a weak point since it restricts the range of domains where iris recognition can be applied, especially those where the subjects' cooperation is not expectable (e.g., criminal/terrorist seek and missing children).

Assuming that, in spite of noise, the iris was accurately segmented, we propose a classification strategy more robust to noise factors. We observed that, in most cases, the noisy data is localized in some of the iris subparts. Our method is based on the division of the segmented iris into six regions, followed by the independent feature extraction in each one. Further, through the comparison between signatures extracted from correspondent iris regions, we obtain six dissimilarity values that are fused through a classification rule. The hope is that most of the iris regions are noise-free and that accurate recognition can be achieved, even in

- The authors are with the Departamento de Informática, Universidade da Beira Interior, Rua Marques D'Avila e Bolama, 6201-001 Covilhã, Portugal.E-mail: \{hugomcp, lfbaa\}@di.ubi.pt.

Manuscript received 1 Feb. 2006; revised 17 July 2006; accepted 6 Nov. 2006; published online 18 Jan. 2007.

Recommended for acceptance by S. Prabhakar, J. Kittler, D. Maltoni, L. O'Gorman, and T. Tan.

For information on obtaining reprints of this article, please send e-mail to: tpami@computer.organd reference IEEECS Log Number TPAMISI-0088-0206. Digital Object Identifier no. 10.1109/TPAMI.2007.1016. highly noisy images. As our experiments confirm, this method decreases-more than 40 percent-the false rejection rates in the recognition of noisy iris images, which can be considered an achievement toward noncooperative iris recognition.

The remainder of this paper is organized as follows: Section 2 briefly summarizes the most cited iris recognition methods and overviews noncooperative iris recognition. A detailed description of the proposed recognition methodology is given in Section 3. Section 4 reports the experiments and discusses the results and, finally, Section 5 presents the conclusions.

\section{IRIS RECOGNITION}

In spite of the specificities of the different proposals, typical iris recognition systems share the structure given in Fig. 1.

The initial stage deals with iris segmentation. This consists of localizing the iris inner (pupillary) and outer (scleric) borders. In 1993, Daugman [6] proposed an integro-differential operator to find both the iris inner and outer borders. Similarly, [7] proposed integro-differential operators that search over the $\mathbb{N}^{3}$ space, with the goal of maximizing the equations that identify the iris borders. Wildes [2] achieved iris segmentation through a gradient-based binary edge map construction followed by circular Hough transform. In [8], the authors proposed a method based on Wildes' method, that, together with a clustering process, achieved robustness for noncooperative environments.

In order to compensate for the variations in the pupil's size and in the imaging distances, it is usual to translate the segmented iris region into a fixed length and dimensionless polar coordinate system. This stage is usually accomplished through the method proposed by Daugman [6].

Regarding feature extraction, iris recognition approaches can be divided into three major categories: phase-based methods (e.g., [6]), zero crossing methods (e.g., [9]), and texture analysis-based methods (e.g., [2]). Daugman [6] uses multiscale quadrature wavelets to extract texture phase information and obtain an iris signature with 2048 binary components. Boles and Boashash [9] computed the zero-crossing representation of a 1D wavelet at different resolutions of concentric circles. Wildes [2] proposes the characterization of the iris texture through a Laplacian pyramid with four different levels (scales).

Last, the comparison between iris signatures is performed, producing a numeric dissimilarity value. If this value is higher than a threshold, the system outputs a nonmatch, meaning that each signature belongs to different irises. Otherwise, the system outputs a match, meaning that both signatures were extracted from the same iris. In this stage, it is common to apply different distance metrics (Hamming [6], Euclidean [10], Weighted Euclidean [11]), or methods based on signal correlation [2].

\subsection{Noncooperative Iris Recognition}

In this paper, we refer to noncooperative iris recognition term as the process of automatically recognizing individuals, using images of their iris captured at-a-distance and without requiring them any active participation. This broadens the iris recognition's applicability to domains where the subjects' cooperation is not expectable (e.g., criminals/terrorists and missing children). However, it is expected that the captured images contain off-angle and partial irises and are blurred (due to bad focus or motion). Some of these noise factors were recently addressed by several authors (e.g., [12] and [13]).

Examples of these and other types of noise are given in Fig. 2. We considered all the types of information other than the iris in the segmented iris ring as noise. According to this definition, there is a 


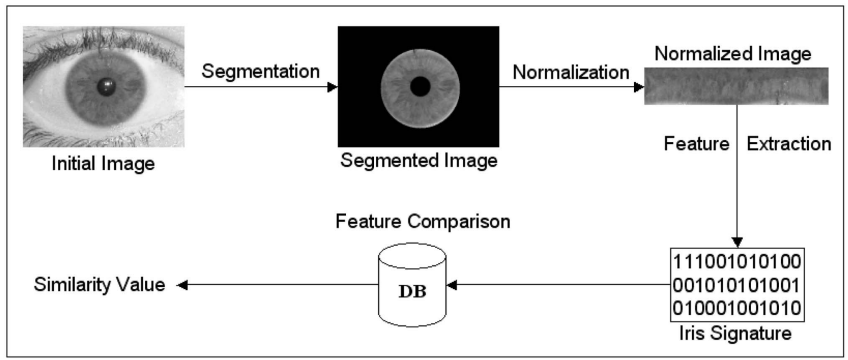

Fig. 1. Typical stages of iris recognition. The process begins with the segmentation of the region correspondent to the iris. To compensate for variations in the pupil's size and in the capturing distance, the image is transformed (normalized) into a dimensionless polar coordinate system. The feature extraction process produces a set of numeric values known as the biometric signature. The comparison between the acquired and the enrolled signatures produces a dissimilarity value used to conclude the identity of the subject.

high probability of capturing images with several noise factors: eyelids (Figs. 2c and 2d), eyelashes (Fig. 2c), glasses, pupil (due to less accurate iris segmentation), motion blur (Fig. 2h), and lighting and specular reflections (Figs. 2e, 2f, and 2g).

\section{Proposed Iris Classification Methodology}

We observed that, in the most cases, the iris images captured in less constrained imaging conditions contain noise that is localized in some of the iris subparts. Moreover, reflections are predominant in the left/right iris extremes and obstructions are usual in the upper and lower parts. Common feature extraction methods focus in the lower and middle-low frequencies of the signal, which, in turn, makes it more probable that noisy data is considered in the creation of the biometric signature.

Previous proposals performed recognition based on small portions of the iris (e.g., [6]), those where noise is less probable. However, they are static and do not take into account the dynamics of the noncooperative imaging environments, which determine the iris regions corrupted by noise.

By dividing the iris into separate regions, our hope is that some of these are noise-free and that the comparison with the respective enrolled region enables accurate recognition. Based on this, in the

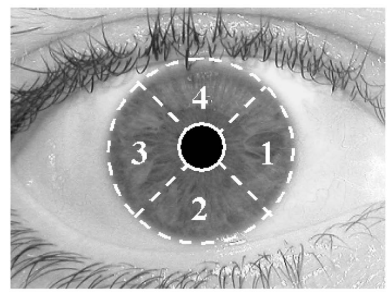

(a)

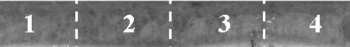

(c)

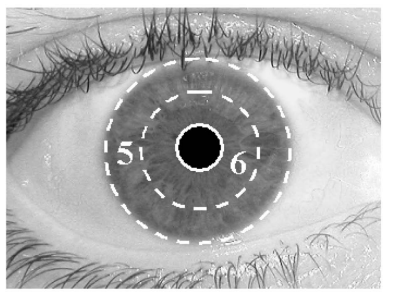

(b)

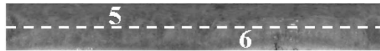

(d)
Fig. 3. Division of the iris into six regions. Making the normalization process as described in [6] and starting from an angle of -45 degrees, regions 1 to 4 correspond to successive quadrants of the iris. Regions 5 and 6 correspond, respectively, to the outer and inner parts of the iris. The main motivation for this division was the observation that the most common types of noise (iris obstructions and reflections) are usual, respectively, in the upper/lower and left/ right portions of the iris. Also, reflections resultant from natural and artificial lighting environments are predominantly localized, respectively, in the outer and inner iris regions. The proposed division strategy minimizes the number of regions simultaneously affected by each type of noise. (a) Division of the iris in four different parts. (b) Division of the iris in "outer" and "inner" parts. (c) Correspondent regions of (a) in the normalized iris image. (d) Correspondent regions of (b) in the normalized iris image.

following sections we describe the proposed iris partition, feature extraction, and classification strategies. As reported in Section 4, this method contributes for a substantial decrease of the error rates in the recognition of noisy iris images.

\subsection{Iris Partition and Feature Extraction}

Through the analysis of the available iris image databases and of our iris imaging experiments, we observed that the most common types of noise (iris obstructions and reflections) are predominantly localized, respectively, in the vertical and horizontal iris extremes. Also, under natural and artificial lighting conditions, reflections are predominant, respectively, in the outer and inner iris regions.

Based on these observations, the proposed iris division schema minimizes the number of regions simultaneously affected by each type of noise, as illustrated in Fig. 3. The independent feature

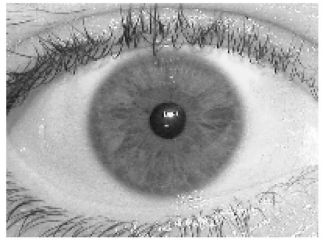

(a)

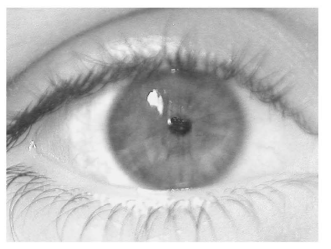

(e)

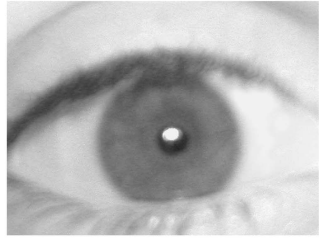

(b)

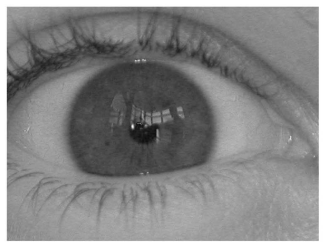

(f)

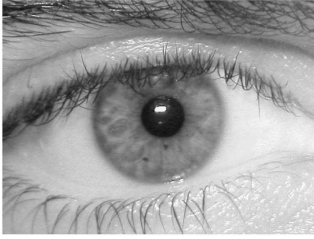

(c)

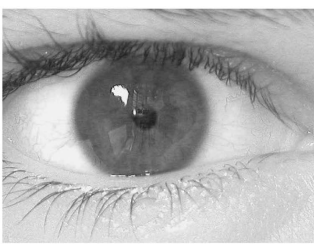

(g)

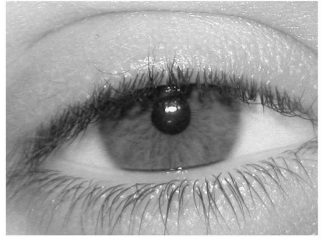

(d)

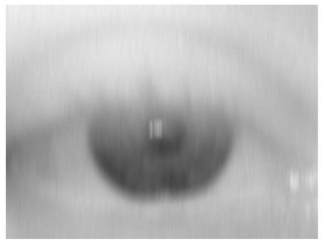

(h)

Fig. 2. Iris images captured under simulated noncooperative conditions. These capture conditions produced images with very heterogeneous characteristics regarding (b) focus, (h) motion blur, contrast, and brightness, as well as (c) and (d) iris occlusions by eyelids or eyelashes. Under natural light environments, captured images will have (e) and $(g)$ specular and (f) and (g) lighting reflections. (a) Good quality iris image. (b) Poorly focused iris image. (c) Iris with eyelids and eyelashes obstruction. (d) Iris with extreme eyelids obstruction. (e) Iris with lighting areas correspondent to reflections from light sources (natural light) next to the user. (f) Iris with specular reflections correspondent to information from the environment that surrounds the user and iris obstruction by the lower eyelid. (g) Iris with specular reflections (region with high intensity in the iris upper left quadrant) and lighting reflections. (h) Motion blurred iris. 
extraction and comparison for each of these regions avoids that the eventual noise, localized in some of the iris subparts, corrupts the whole biometric signature.

According to the chosen feature extraction method, the process consists of the creation of six independent biometric signatures, each one correspondent to a specific iris region. The details of the tested feature extraction method are given in Section 4. However, we stress that this process is independent of the feature extraction strategy. Oppositely, as starts from the normalized iris image, it is dependent of the previous accurate iris segmentation, which is obviously a challenge, given the dynamics of the noncooperative image capturing environments.

\subsection{Feature Comparison and Classification}

In the following discussion, we will use a superscript to distinguish between two different iris images, such as $I^{1}$ and $I^{2}$, and a subscript to distinguish between different regions of an iris image, such as $I_{1}^{1}$ and $I_{2}^{1}$.

Let $I_{j}$ be a region of an iris image, such as the ones identified in Fig. 3. We define the result of the function $s\left(I_{j}\right): I_{j} \rightarrow \mathbb{R}^{k}$ as the biometric signature of $I_{j}$. Next, we define the dissimilarity between two signatures as $d\left(s\left(I_{j}^{1}\right), s\left(I_{j}^{2}\right)\right): \mathbb{R}^{k} \times \mathbb{R}^{k} \rightarrow \mathbb{R}$, noting that these values are found for two signatures extracted from correspondent iris regions.

If we choose to define $N$ regions in an iris image $I_{i}, i=1, \ldots, N$, we obtain $N$ signatures $s\left(I_{i}\right)$, one for each region. Consider $I^{1}$ and $I^{2}$, respectively, as the template and sample images. For $I^{1}$, we have the $N$ signatures $\left\{s\left(I_{1}^{1}\right), \ldots, s\left(I_{N}^{1}\right)\right\}$ and, for $I^{2}$, the correspondent $\left\{s\left(I_{1}^{2}\right), \ldots, s\left(I_{N}^{2}\right)\right\}$.

Let $D$ be the set that contains the dissimilarities between the correspondent regions of $I^{1}$ and $I^{2}: D=\left\{D_{1}, \ldots, D_{N}\right\}$, where $D_{i}=d\left(s\left(I_{i}^{1}\right), s\left(I_{i}^{2}\right)\right), i=1, \ldots, N$.

Let $T=\left\{T_{1}, \ldots, T_{N}\right\}, T_{i} \in \mathbb{R}^{+}$, be a set of $N$ threshold values, such that $T_{i} \leq T_{j}, \forall i<j$.

We define the function $C\left(D, T_{i}\right): \mathbb{R}^{N} \times \mathbb{R}^{+} \rightarrow \mathbb{N}$, that counts the number of $D_{j} \in D$ that are smaller or equal to $T_{i}$, as:

$$
C\left(D, T_{i}\right)=\sum_{j=1}^{N} \mathbb{I}_{\left\{D_{j} \leq T_{i}\right\}},
$$

where $\mathbb{I}_{\{.\}}$is the indicator function. iris if:

Images $I^{1}$ and $I^{2}$ are classified as corresponding to the same

$$
\exists_{i}: C\left(D, T_{i}\right) \geq i, i=1, \ldots, N .
$$

The rationale behind this classification strategy is the inverse correspondence between the number of comparisons that must be smaller or equal to $T_{i}$ and the constraining degree of $T_{i}$. Fig. 4 illustrates three examples of the application of the proposed classification strategy. It contains the dissimilarities between the template and the sample biometric signatures of three subjects, represented in the horizontal axis (1,2, and 3). The similarity threshold set $(T)$ is represented by the horizontal dashed lines. The application of (2) confirms the identity of subjects 1 and 2 and denies the identity of subject 3 . Subject 1 has one dissimilarity value below the lower threshold $\left(T_{1}\right)$ and subject 2 has two dissimilarities below the second lower threshold $\left(T_{2}\right)$.

As detailed in Section 4.3, an exhaustive search is used to select an optimal set of thresholds $(T)$. As our experiments report, this method significantly decreases the false-rejection rates in the recognition of noisy iris images since it isolates the noisy data in the correspondent signature, extracted from the iris region that contains it. This decreases the probability that noisy iris regions corrupt the whole biometric signature when extracting the lower frequency components of the signal.

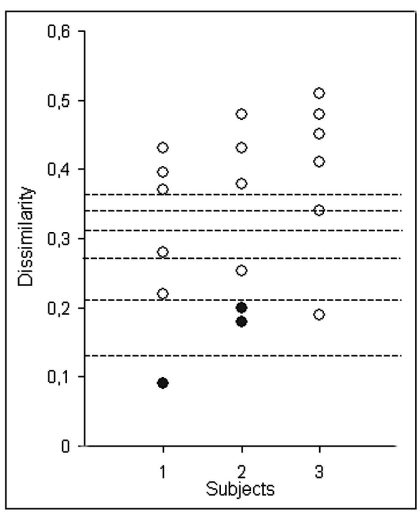

Fig. 4. Examples of the classification process. The horizontal axis represents the subjects $(1,2$, and 3$)$ and the vertical axis the dissimilarity values between the correspondent template and the sample signatures. The threshold set $(T)$ is represented by the horizontal dashed lines. The application of (2) confirms the identity of subjects 1 and 2 since subject 1 has one dissimilarity below the lower threshold and subject 2 has two dissimilarities below the second lower threshold, whereas denies the identity of subject 3 since it just has two dissimilarities below the upper threshold. The dissimilarities that determined the identity acceptance are represented by filled circles, oppositely to the remaining ones.

However, if the iris is divided as described above, it is not possible to achieve invariance to iris rotation through signature shifting, and this is a very common technique used in feature comparison. We compensate for this by making the normalization process into the dimensionless polar coordinate system starting from five different deviation angles of the segmented iris image $(-10,-5,0,+5$, and +10 degrees) and obtaining five normalized iris images. The subsequent processing is further made separately for each of the images, and the final similarity is given by the highest similarity between the enrolled signatures and those extracted from each image.

\section{EXPERIMENTS AND RESULTS}

To enable the effective test of the proposed classification strategy, we analyzed the available iris databases and selected the most appropriate for our purposes. In the following section, we briefly describe the available public iris image databases and the data sets used in our experiments.

\subsection{Iris Databases}

There are presently seven public and freely available iris image databases for biometric purposes: CASIA [14], MMU [15], BATH [16], UPOL [17], ICE [18], WVU [19], and UBIRIS [20].

The CASIA database is by far the most widely used for iris biometric purposes. However, its images incorporate few types of noise, almost exclusively related with eyelid and eyelash obstruction, similarly to the images from MMU and BATH databases. UPOL images were captured with an optometric framework, obtaining optimal images with extremely similar characteristics. Although ICE and WVU databases contain images with more noise factors, their lack of images with significant reflections within the iris rings constitutes a weak point regarding the simulation of noncooperative imaging conditions. Oppositely, images of the UBIRIS database were captured under natural lighting and heterogenous imaging conditions, which explains their higher heterogeneity.

Based on the manual verification of the iris segmentation accuracy in each of the images, we selected 800 images from 80 subjects of the UBIRIS database. In order to evaluate the recognition accuracy both in highly and less noisy environments, an equal number of images from the CASIA and ICE databases were 


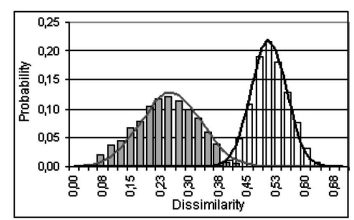

(a)

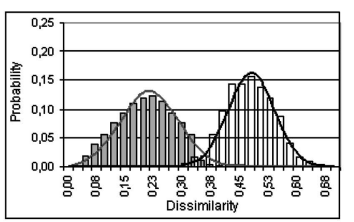

(b)

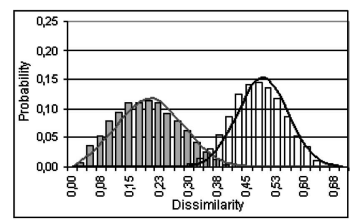

(c)

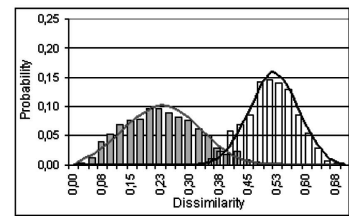

(d)

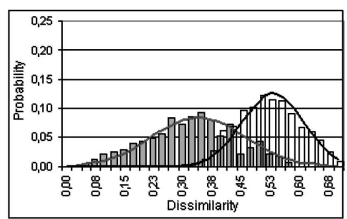

(e)

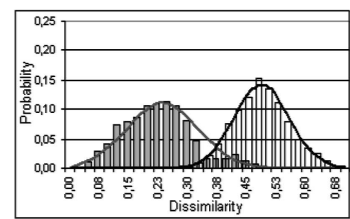

(f)

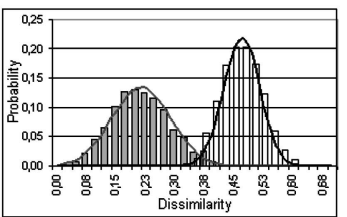

(g)

Fig. 5. Histograms of the dissimilarities between the signatures extracted from the (a) whole iris and each of the regions identified in Fig. 3 . These values correspond to the $U B I R I S_{t r}$ data set and the line plots approximate normal distributions obtained through curve fitting. We concluded that each of the isolated regions can distinguish between individuals since a clear separability between the intra and interclass comparisons can be observed, when using each of the iris regions exclusively. (a) Whole iris distribution. $\tau_{W}=78.3$. (b) Region "1" distribution. $\tau_{1}=76.0$. (c) Region "2" distribution. $\tau_{2}=74.7$. (d) Region "3" distribution. $\tau_{3}=66.1$. (e) Region "4" distribution. $\tau_{4}=39,2$. (f) Region "5" distribution. $\tau_{5}=56,3$. (g) Region "6" distribution. $\tau_{6}=72,5$.

selected, having, once again, manually verified the accuracy of the iris segmentation. Further, we divided each data set into two halves. The first data sets- $U B I R I S_{t r}, C A S I A_{t r}$, and $I C E_{t r}$-were used as training sets, to obtain the threshold sets $(T)$, and the later-UBIRIS $S_{t t}, C A S I A_{t t}$, and $I C E_{t t}$ - to evaluate the recognition accuracy.

Each data set enables, respectively, 1,800 and 78,000 intra and interclass comparisons. Images of the UBIRIS data sets contain iris obstructions by eyelids and eyelashes, poor focused and motion blurred irises, and irises with specular and lighting reflections, while those of the CASIA and ICE data sets contain, almost exclusively, iris obstructions by eyelids and eyelashes and a small number of poorly focused images.

\subsection{Description of Experiments}

We implemented the recognition method described by Daugman [6] and compared the obtained results when following the method as described by the author and using the proposed iris division and classification strategies. Initially, we made the feature extraction and comparison using the whole segmented iris, extracting a total of 2,048 bits. Further, according to Fig. 3, we divided the iris into six regions and, through feature extraction, obtained 512 and 1,024 bits, respectively, for the signatures extracted from the iris regions 1 to 4 and 5 to 6 . The iris classification was made through the fusion rule given by (2). We stress that we did not implement any noise detection technique, which increased the error rates but enabled clear conclusions about the robustness to noise of each classification method.

As described in [6], the Daugman's recognition method is divided into the following stages:

- Iris segmentation. We implemented the integrodifferential operator proposed by the author to find both the inner and outer iris borders, given by

$$
\max _{r, x_{0}, y_{0}}\left|G_{\sigma}(r) * \frac{\delta}{\delta r} \oint_{r, x_{0}, y_{0}} \frac{I(x, y)}{2 \pi r} d s\right|
$$

This operator searches over the image domain $(x, y)$ for the maximum in the blurred partial derivative with respect to increasing radius $r$ of the normalized contour integral of $I(x, y)$ along a circular arc $d s$ of radius $r$ and center coordinates $\left(x_{0}, y_{0}\right)$.
- Normalization. After the segmentation of both iris borders, to compensate for the varying size of th pupil and capturing distance, we translated the images into a dimensionless polar coordinate system, according to the process known as the Daugman Rubber Sheet [6].

- Feature Extraction. The iris data encoding was accomplished through the use of two-dimensional Gabor filters. These spatial filters have the form $G(x, y)=e^{-\pi \Phi} \cdot e^{-2 \pi i \Psi}$, with

$$
\Phi=\left[\left(x-x_{0}\right)^{2} / \alpha^{2}+\left(y-y_{0}\right)^{2} / \beta^{2}\right]
$$

and

$$
\Psi=\left[u_{0}\left(x-x_{0}\right)+v_{0}\left(y-y_{0}\right)\right] .
$$

$\left(x_{0}, y_{0}\right)$ defines the position in the image, $(\alpha, \beta)$ is the filter width and length and $\left(u_{0}, v_{0}\right)$ specifies the modulation, having spatial frequency $w_{0}=\sqrt{u_{0}^{2}+v_{0}^{2}}$ and direction $\theta_{0}=\arctan \left(v_{0} / u_{0}\right)$. To achieve invariance to illumination, the real parts of the 2D Gabor filters were truncated to be zero volume and the sign of the real and imaginary parts from quadrature image projections quantized into binary values.

- Feature Comparison. The binary features allowed the use of the Hamming distance as the similarity measure between two iris signatures. Given two binary sets with $N$ bits: $A=\left\{a_{1}, \ldots, a_{N}\right\}$ and $B=\left\{b_{1}, \ldots, b_{N}\right\}$, the Hamming distance is given by $H D(A, B)=\frac{1}{N} * \sum_{i=1}^{N} a_{i} \otimes b_{i}$, where $a \otimes b$ is the logical XOR operation.

\subsection{Thresholds and Performance}

The proposed classification strategy requires the computation of the optimal dissimilarity threshold set $(T)$ that minimizes the error rates. In order to obtain it, we started by the feature extraction and comparison using the whole iris and each of the proposed regions (exclusively) in the training data sets.

Fig. 5 contains the histograms of the dissimilarities between the signatures extracted from the $U B I R I S_{t r}$ data set, using the whole iris (Fig. 5a) and each of the regions individually (Figs. 5b, 5c, 5d, 5e, $5 \mathrm{f}$, and $5 \mathrm{~g}$ ). The lines correspond to the approximated normal distributions obtained through curve fitting, with a 95 percent confidence interval. $\tau_{i}$ contains the value for a t-test given by: 


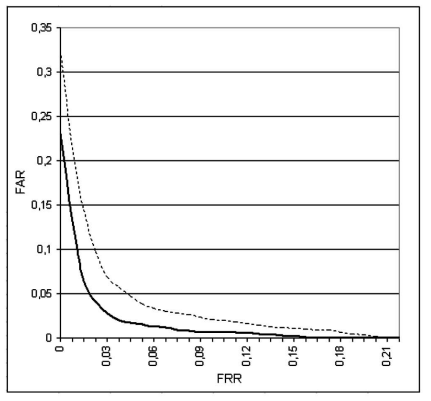

(a)

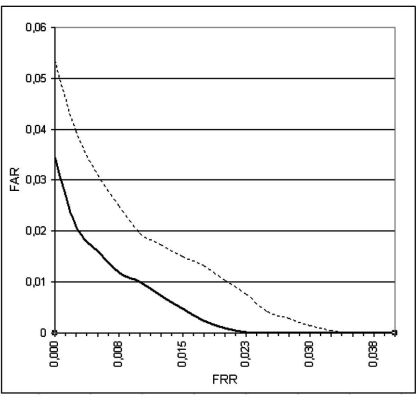

(b)

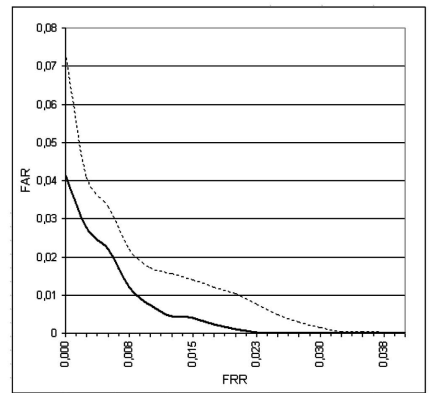

(c)

Fig. 6. Comparison between the ROCs obtained by the classical Daugman recognition method (dashed lines) and our iris division and classification proposals (continuous lines) in the (a) $U B I R I S_{t t}$, (b) $C A S I A_{t t}$, and (c) $I C E_{t t}$ data sets. Notice the different scales in the figures and the larger errors in the noisier $U B I R I S_{t t}$ data set. (a) ROCs obtained in the $U B I R I S_{t t}$ data set. (b) ROCs obtained in the $C A S I A_{t t}$ data set. (c) ROCs obtained in the $I C E_{t t}$ data set.

$$
\tau_{i}=\frac{\mu^{E}-\mu^{I}}{\sqrt{\frac{\sigma^{I^{2}}}{N^{I}}+\frac{\sigma^{E^{2}}}{N^{E}}}},
$$

where $\mu^{I}$ and $\mu^{E}$, respectively, indicate the mean of the intra and interclass dissimilarities. $\sigma^{I}$ and $\sigma^{E}$ indicate the respective standard deviations and $N^{I}$ and $N^{E}$ are, respectively, the number of intra and interclass comparisons.

Through this process, we obtained the parameters of the normal distributions for each of the iris regions and type of comparisons (intra and interclass). Let the subscript denote the image regions identified in Fig. $3(1, \ldots, 6)$ and the superscript denote, respectively, the intra (I) and interclass (E) comparisons, such as $N_{1}^{I}$ (the normal distribution of the intraclass comparisons between the irises region 1).

The goal is to find the values for the ordered threshold set $T=$ $\left\{T_{1}, \ldots, T_{6}\right\}$ that minimize the error rates. Let $D^{E}=\left\{D_{1}, \ldots, D_{6}\right\}$ be the set of random variables following, respectively, the $N_{i}^{E}, i=$ $1, \ldots, 6$ distributions. According to (1), $C\left(D^{E}, T_{k}\right)$ is the function that counts the number of $D_{j} \in D^{E}$ that are smaller or equal to $T_{k}$. The FAR is given by:

$$
F A R=\sum_{i=1}^{6} P\left(C\left(D^{E}, T_{i}\right) \geq i \mid \bigcap_{j=1}^{i-1} C\left(D^{E}, T_{j}\right)<j\right),
$$

where $P(A \mid B)$ denotes the probability of $A$ conditioned by the occurrence of $B$. Using the same notation, let $D^{I}=\left\{D_{1}, \ldots, D_{6}\right\}$ be the set of random variables following, respectively, the $N_{i}^{I}, i=$ $1, \ldots, 6$ distributions. The FRR is given by:

$$
F R R=\prod_{i=1}^{6} P\left(C\left(D^{I}, T_{i}\right)<i\right)
$$

Using (3) and (4), we made an exhaustive search in the $[0,1]$ interval, having tested all the possible combinations for the six thresholds values with $10^{-3}$ precision.

Fig. 6 contains the obtained receiver operating curves (ROCs) when following the classical Daugman recognition method (dashed lines) and our proposal (continuous lines) in the $U B I R I S_{t t}$, $C A S I A_{t t}$, and $I C E_{t t}$ data sets. These results show that our proposal consistently decreased the error rates, either in the highly noisy $\left(U B I R I S_{t t}\right)$ or less noisy images $\left(C A S I A_{t t}\right.$ and $\left.I C E_{t t}\right)$.

\subsection{Discussion}

Table 1 contains a comparison of the obtained results by the original classification method, as described by Daugman, our iris division and classification proposal, and three common classification fusion strategies (mean, minimum, and product). The first column identifies the classification method, and the second contains the false rejection rates when preventing the false accept errors. EER corresponds to the equal error rate and, finally, the last column contains the approximate percent value of the area under the ROC, which corresponds to the error area. All the error rates are expressed for a confidence interval of 95 percent.

We observed that our method consistently achieved better performance when compared to the original proposal. These can be observed either in the highly noisy images from the UBIRIS database or in the less noisy images from the CASIA and ICE databases.

This higher accuracy in the recognition of noisy images can be explained by its ability to perform recognition using small portions of the iris, avoiding the corruption of the whole signature by localized noisy regions. These results led us to conclude that the proposed iris division and classification strategy is more tolerant to noise factors and, for this reason, is more adequate for the application is less constrained imaging environments.

\section{Conclusions}

In this paper, we addressed the problems motivated by the existence of noise in the captured iris images and the correspondent increase of the error rates, with particular relevance to the false rejections, in the context of noncooperative iris recognition.

Common feature extraction proposals usually focus on the lower and middle-low frequency components of the signal. This implies that small portions of nondetected noise can corrupt the whole biometric signature and decrease the recognition accuracy.

TABLE 1

Comparison between the Error Rates Obtained When Following the

\begin{tabular}{|c|c|c|c|}
\hline Classification Strategy & $F R R, F A R=0(\%)$ & $E E R(\%)$ & Error $R O C(\%)$ \\
\hline \multicolumn{4}{|l|}{$U B I R I S_{t t}$ data set } \\
\hline Proposed & $16.49 \pm 0.01$ & $2.38 \pm 0.00$ & 1.73 \\
\hline Daugman original & $22.95 \pm 0.02$ & $3.72 \pm 0.00$ & 3.21 \\
\hline Mean & $19.90 \pm 0.01$ & $4.98 \pm 0.00$ & 3.30 \\
\hline Minimum & $18.38 \pm 0.01$ & $4.80 \pm 0.00$ & 2.39 \\
\hline Product & $18.81 \pm 0.01$ & $4.81 \pm 0.00$ & 2.81 \\
\hline \multicolumn{4}{|l|}{$C A S T A_{t t}$ data set } \\
\hline Proposed & $2.39 \pm 0.00$ & $1.01 \pm 0.00$ & 0.55 \\
\hline Daugman original & $3.41 \pm 0.00$ & $1.44 \pm 0.00$ & 0.64 \\
\hline Mean & $3.11 \pm 0.00$ & $1.26 \pm 0.00$ & 0.61 \\
\hline Minimum & $4.62 \pm 0.00$ & $1.29 \pm 0.00$ & 0.64 \\
\hline Product & $2.95 \pm 0.00$ & $1.20 \pm 0.00$ & 0.60 \\
\hline \multicolumn{4}{|l|}{$I C E_{t t}$ data set } \\
\hline Proposed & $2.30 \pm 0.00$ & $1.03 \pm 0.00$ & 0.72 \\
\hline Daugman original & $3.78 \pm 0.00$ & $1.71 \pm 0.00$ & 0.99 \\
\hline Mean & $3.63 \pm 0.00$ & $1.26 \pm 0.00$ & 1.33 \\
\hline Minimum & $3.65 \pm 0.00$ & $1.26 \pm 0.00$ & 1.42 \\
\hline Product & $3.98 \pm 0.00$ & $1.31 \pm 0.00$ & 1.65 \\
\hline
\end{tabular}
Original Daugman's Recognition Method, Our Classification Strategy, and Three Common Classification Fusion Strategies 
Based on this, we proposed a new iris classification strategy that divides the segmented and normalized iris into six regions and makes an independent feature extraction and comparison for each of these regions. Iris classification is achieved through a fusion rule that uses a threshold set to combine the dissimilarity values resultant from the comparison between correspondent iris regions.

Experiments in the recognition of noisy images showed a significant decrease in the error rates, about 30 percent in the false rejections $(F R R, F A R=0$ from 22.95 to 16.49 percent, 3.41 to 2.39 percent, and 2.30 to 3.78 percent, respectively, in the UBIRIS, CASIA, and ICE data sets) when compared to Daugman's original iris classification proposal. Moreover, as can be seen by the results presented in Table 1, we compared the accuracy of our proposal with three common classification fusion strategies-product, mean, and minimum - and the proposed method consistently achieved lower error rates. This indicates that the proposed method is adequate for less constrained image capturing environments, such as in a noncooperative setting, and broadens the range of domains where iris recognition can be applied.

However, we stress that these results are dependent on the previous accurate iris segmentation, which is highly challenging, given the dynamics of noncooperative environments. The requirement of optical frameworks that are able to capture iris images with enough quality and of real-time face and eye localization methods is assumed too.

\section{REFERENCES}

[1] L. Flom and A. Safir, "Iris Recognition System," US Patent 4641 394, 1987.

[2] R.P. Wildes, "Iris Recognition: An Emerging Biometric Technology," Proc. IEEE, vol. 85, no. 9, pp. 1348-1363, Sept. 1997.

[3] L. Ma, T. Tan, D. Zhang, and Y. Wang, "Local Intensity Variation Analysis for Iris Recognition," Pattern Recognition, vol. 37, no. 6, pp. 1287-1298, 2004.

[4] M. Vatsa, R. Singh, and A. Noore, "Reducing the False Rejection Rate of Iris Recognition Using Textural and Topological Features," Int'l J. Signal Processing, vol. 2, no. 1, pp. 66-72, 2005.

[5] "Independent Test of Iris Recognition Technology," Int'l Biometric Group, http://www.biometricgroup.com/reports, 2005.

[6] J.G. Daugman, "High Confidence Visual Recognition of Persons by a Test of Statistical Independence," IEEE Trans. Pattern Analysis and Machine Intelligence, vol. 25, no. 11, pp. 1148-1161, Nov. 1993.

[7] T. Camus and R. Wildes, "Reliable and Fast Eye Finding in Close-Up Images," Proc. IEEE 16th Int'l Conf. Pattern Recognition, pp. 389-394, Aug. 2002.

[8] H. Proença and L.A. Alexandre, "Iris Segmentation Methodology for Noncooperative Iris Recognition," IEE Proc. Vision, Image, and Signal Processing, vol. 153, no. 2, pp. 199-205, Apr. 2006.

[9] W.W. Boles and B. Boashash, "A Human Identification Technique Using Images of the Iris and Wavelet Transform," IEEE Trans. Signal Processing, vol. 46, no. 4, pp. 1185-1188, Apr. 1998.

[10] Y. Huang, S. Luo, and E. Chen, "An Efficient Iris Recognition System," Proc. First Int'l Conf. Machine Learning and Cybernetics, pp. 450-454, Nov. 2002.

[11] L. Ma, Y. Wang, and T. Tan, "Iris Recognition Using Circular Symmetric Filters," Proc. 25th Int'l Conf. Pattern Recognition, vol. 2, pp. 414-417, Aug. 2002.

[12] V. Dorairaj, N. Schmid, and G. Fahmy, "Performance Evaluation of Nonideal Iris Based Recognition System Implementing Global ICA Encoding," Proc. IEEE Int'l Conf. Image Processing, pp. 285-288, Sept. 2005.

[13] N. Kalka, J. Zuo, N. Schmid, and B. Cukic, "Image Quality Accessment for Iris Biometric," Proc. SPIE Conf. Biometric Technology for Human Identification III, vol. 6202, pp. 263-273, Apr. 2006.

[14] "CASIA Iris Image Database," Inst. of Automation, Chinese Academy of Sciences, http://www.sinobiometrics.com, 2004.

[15] "MMU Iris Image Database," Multimedia Univ., http://pesona. mmu.edu.my/ccteo, 2004.

[16] "University of Bath Iris Image Database," Univ. of Bath, http:// www.bath.ac.uk/elec-eng/pages/sipg/, 2004.

[17] M. Dobes and L. Machala, "UPOL Iris Image Database," http:// phoenix.inf.upol.cz/iris/, 2004

[18] "Iris Challenge Evaluation," Nat'l Inst. of Standards and Technology, http:/ /iris.nist.gov/ICE/, 2006.

[19] A. Ross, S. Crihalmeanu, L. Hornak, and S. Schuckers, "A Centralized WebEnabled Multimodal Biometric Database," Proc. 2004 Biometric Consortium Conf., Sept. 2004.

[20] H. Proença and L.A. Alexandre, "UBIRIS: A Noisy Iris Image Database," Proc. 13th Int'l Conf. Image Analysis and Processing, pp. 970-977, Sept. 2005, http:/ /iris.di.ubi.pt.

$\triangleright$ For more information on this or any other computing topic, please visit our Digital Library at www.computer.org/publications/dlib. 